\title{
Plasma-based active closed-loop control of instability waves in unexcited turbulent jet. Part 2. Installed jet.
}

\author{
Oleg Bychkov ${ }^{1,2 *}$, Georgy Faranosov ${ }^{1,2 \dagger}$, Victor Kopiev ${ }^{1 \ddagger}$, Vladimir Kopiev ${ }^{1 \S}$, \\ ${\text { Ivan } \text { Moralev }^{3 * *} \text {, Pavel Kazansky }}^{3 \dagger \dagger}$ \\ ${ }^{1}$ Central Aerohydrodynamic Institute (TsAGI), Moscow, Russia, 105005 \\ ${ }^{2}$ Moscow Institute of Physics and Technology, Zhukovsky, Russia, 140180 \\ ${ }^{3}$ Joint Institute of High Temperature RAS, Moscow, Russia, 125412
}

\begin{abstract}
The paper considers the possibility of controlling jet-wing interaction noise based on the results obtained for free jet in the Part I of the study. ${ }^{1}$ The main idea is connected with the control of the amplitude of wave packets developing in a turbulent jet. The presence of a wing makes diffraction of the jet near-field pulsations an effective sound source in case of close location of the jet shear layer and the wing trailing edge. Therefore, a decrease in the intensity of wave packets can lead to noise reduction in the far field of the installed jet. In the current work it is shown, for the first time, that active closed-loop control system using high frequency dielectric barrier discharge (HF DBD) plasma actuator can lead to a reduction of pressure pulsations not only in the near-field but also in the far acoustic field of the jet. Initially, the effect of the installation noise reduction is demonstrated for an excited jet. Then, the possibility of noise reduction of unexcited installed jet is shown. The location of the actuator on the edge, the required energy input, and the robustness of the control system itself are quite promising in terms of noise reduction of realistic jet-wing configurations.
\end{abstract}

\section{Introduction}

$\mathrm{E}_{\mathrm{s}}^{\mathrm{N}}$ NGINE remains one of the most significant contributors to community noise levels of civil aircrafts. New generation engines used on modern airplanes typically have high or ultra-high bypass ratios and a correspondingly larger diameter so that they are located rather close to the aircraft wing. It is well known ${ }^{2-5}$ that the noise of a jet located close to a wing can be significantly changed compared to that of a free jet. Particularly, at low and moderate frequencies, it may cause the occurrence of an additional efficient noise source associated with the interaction between the hydrodynamic near field of the jet and the wing trailing edge. ${ }^{5-17}$

To reduce jet-wing installation noise, several approaches can be used. Most popular methods are related to the modifications of nozzle-wing geometry: nozzle modifications or wing/flaps modifications. ${ }^{3,4,7,10,12}$ However, changing of the baseline geometry may not be optimal from aerodynamic point of view. Therefore, development of noise reduction technologies, not requiring geometrical modifications, is valuable. One of the possibilities for the realization of such kind of methods is to use active flow control systems.

While the problem of active control of free (uninstalled) jet noise has a rich background, ${ }^{18-30}$ installed jet noise, to our knowledge, has not been considered from this point of view. Recent studies ${ }^{5,6,9-11,13-17}$ have revealed that significant noise intensification at low and moderate frequencies observed for installed jets is related to the scattering of the jet near-field pressure pulsations on the wing trailing edge. This pressure field is associated with instability waves developing in the jet shear layer. ${ }^{31-34}$ Therefore, instability wave control can also be regarded as universal noise reduction approach for jet-wing configurations as was proposed by Belyaev et al. ${ }^{34}$

This work is devoted to the elaboration and experimental testing of the plasma-based active closed-loop system to control instability waves, and hence noise of an installed jet. The approach used is based on the results of the

\footnotetext{
* PhD Student, Junior Researcher, Acoustic Department

$\dagger$ PhD, Leading Researcher, Acoustic Department

$\$$ Professor, Head of Acoustic Department, vkopiev@mktsagi.ru, AIAA Member

${ }^{\S} \mathrm{PhD}$, Leading Researcher, Acoustic Department

** $\mathrm{PhD}$, Head of Department, Department of plasma aerodynamics and stimulated combustion

${ }^{\dagger \dagger} \mathrm{PhD}$, Senior Researcher, Department of plasma aerodynamics and stimulated combustion
} 
Part I of the study ${ }^{1}$ where the concept, initially described in Belyaev et al., ${ }^{34}$ was realized. In the present work, the system developed in Bychkov et al. ${ }^{1}$ is extended to the case of the installed jet and its performance is tested in experiments with a simplified jet-plate configuration.

The paper is organized as follows. In section II, the control strategy is presented. Section III describes experiments with an excited installed jet and it is devoted to the general proof of concept on the installed jet noise control via instability waves suppression. Experimental setup and the results of noise control for the unexcited jet are presented in Section IV. Concluding remarks are given in Section V.

\section{Control strategy}

The main idea of the control of the low-frequency installation noise is based on the assumption about the dominant role of instability waves in the process of noise generation..$^{6,9-11,13-17}$ It was shown ${ }^{6,14,15}$ that if the trailing edge of the wing lies in the irrotational near field of the jet, the problem of the low-frequency installation noise modeling can be solved in a linear framework. Namely, for each frequency, there is a linear transfer function between the near-field hydrodynamic pulsations of the jet, considered as if the plate is absent, and the far-field sound caused by the scattering of these pulsations on the trailing edge:

$$
p_{m}(\vec{R}, \omega)=p_{m}^{\prime}(\omega) F_{m}(\vec{R}, \omega),
$$

where $p_{m}^{\prime}$ is the pressure of the $m$-th azimuthal mode in the location of the trailing edge (TE), $p_{m}$ is the far-field acoustic pressure due to the scattering of the $m$-th near-field azimuthal mode, and $F_{m}$ is the transfer function between the two. Detailed description of the functions may be found in Bychkov \& Faranosov. ${ }^{15}$ The value $p_{m}^{\prime}$ is mainly determined by the instability wave packets of the corresponding azimuthal order. It was shown by Lyu et al. ${ }^{14}$ and Bychkov \& Faranosov ${ }^{15}$ that the two first azimuthal modes mainly contribute to the low-frequency part of the installation noise. Therefore, if the control system is capable to reduce instability waves of the azimuthal orders $m=0$ and/or $m=1$, it should also lead to the reduction in installation noise. This anticipated effect is qualitatively sketched in Fig. 1.

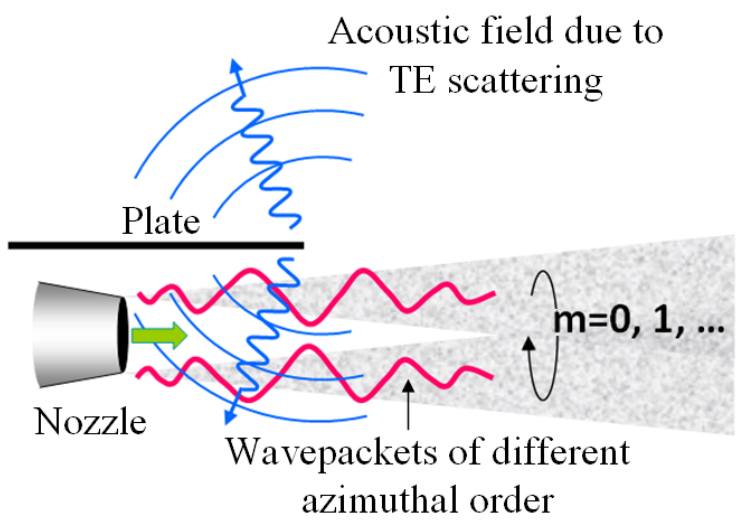

(a)

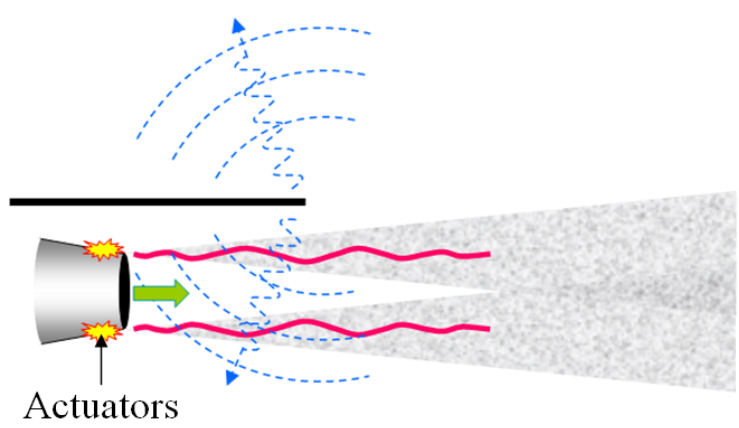

(b)

Figure 1. Main idea of the installation noise control. (a) - uncontrolled jet; (b) - controlled jet.

In the current work, we propose to control instability waves by means of plasma actuators mounted inside the nozzle near its edge (Fig. 1). This approach was successfully tested for uninstalled jets both for artificially excited instability waves (AIW) ${ }^{24-26}$ and natural instability waves (NIW). ${ }^{1}$ The control system is constructed on basis of the concept developed in Belyaev et al. ${ }^{34}$ and implemented for uninstalled jets in Bychkov et al. ${ }^{1}$ The proposed control strategy consists in the narrowband sliding filtration of the original signal and the formation of the corresponding narrowband control action. The strategy relies on the linear principle of signal superposition. It is assumed that if the control action generates instability waves in antiphase to the natural ones and of the same amplitude, then the total signal will be reduced, and, in accordance with the abovementioned physical mechanism, installation noise should be also attenuated. 


\section{Proof of concept: control of installation noise of excited jet}

\section{A. Experimental setup}

The goal of the first test campaign reported in this work was to confirm the acoustic effect of instability wave control in an installed configuration Experiments were carried out in TsAGI anechoic chamber AC-2. Round conical nozzle of diameter $D=60 \mathrm{~mm}$ was used together with an aluminum rectangular plate of dimensions $1.2 \mathrm{~m} \times 0.35 \mathrm{~m} \times 0.003 \mathrm{~m}$. A photo of the setup can is shown in Fig. 2. Relative position of the nozzle and the plate is defined by $h=0.8 D, l=D$ (Fig. 3). The rationale for this plate location is given below. The measurements were performed at exit velocity corresponding to acoustic Mach number $M_{j}=0.4$. View of the setup is shown in Fig. 2 . The jet could be excited by acoustic or/and plasma actuators described below.

Artificial instability waves in the jet were generated by acoustic excitation. It was implemented in a similar as in Kopiev et al. $^{24}$ A loudspeaker was installed in the stagnation chamber about $1.5 \mathrm{~m}$ upstream from the nozzle exit (Fig. 3). The cut-off frequency of the exhaust duct (of about $1 \mathrm{~m}$ length) was above $5 \mathrm{kHz}$. Excitation frequencies used for the current study were well below this value ensuring that only plane acoustic wave could propagate to the nozzle exit. The loudspeaker was connected to the generator output of the PULSE 3560D data acquisition system. The frequency of the excitation was defined by the generator settings, while its amplitude was controlled by the external amplifier.

Control signal was generated by a high frequency dielectric barrier discharge (HF DBD) plasma actuator mounted on the inner surface of the nozzle (Fig. 2b). Such kind of actuator was used previously in the control system to suppress artificially excited instability waves (AIW) in free jet. ${ }^{26}$ Unlike the work of Kopiev et al., ${ }^{26}$ where specially manufactured ceramic nozzles were used, in the current study we have used metal nozzles with covered by ceramic coating $1 \mathrm{~mm}$ thick. Such a technology makes it possible to use existing metal nozzles and thus is more convenient than the previous one. ${ }^{26}$ The discharge was created on the edges of the ring metal strip, and the nozzle itself served as the second electrode (Fig. 2b). The distance from the edge of the electrode to the nozzle exit plane was within 3-5 mm (Fig. 2b). Actuator was powered by the sine voltage with amplitude $5-10 \mathrm{kV}$ and frequency $\sim 200 \mathrm{kHz}$. More detailed description of the actuator may be found in Bychkov et al. ${ }^{1}$

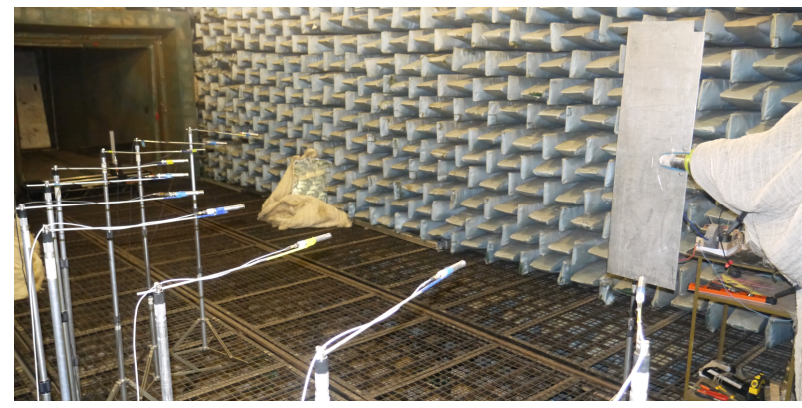

(a)

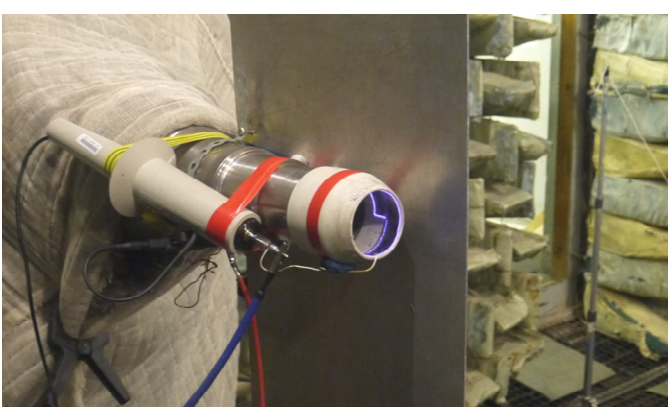

(b)

Figure 2. Photograph of the test setup. (a) - general view; (b) - installed nozzle with plasma actuator.

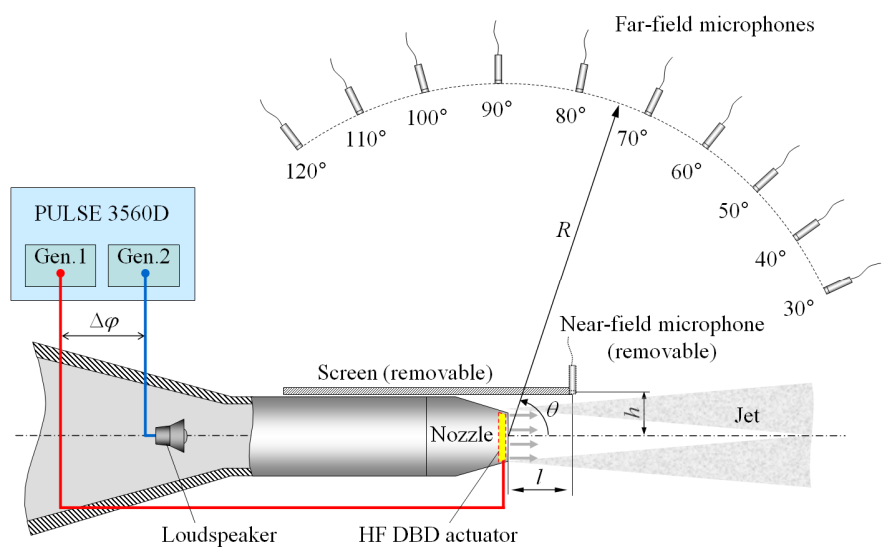

Figure 3. Sketch of the setup for the excited jet investigation. 
Far-field measurements were performed by 10 Bruel \& Kjaer 1/2” 4189 microphones with 2669 Bruel \& Kjaer preamplifiers. The microphones were located on a circular arc at a radial distance $R=33.3 \mathrm{D}$ from the nozzle exhaust and spanned a polar angle range from $\theta=30^{\circ}$ to $120^{\circ}$ as shown in Fig. 3. The polar angle $\theta=0^{\circ}$ corresponded to an observer position downstream of the nozzle on the jet axis. All of the measurements were repeated for the same operating conditions for isolated and installed configurations, so that the microphone arc was on the shielded side of the jet (Fig. 3).

Adjustment of the control parameters was performed for the uninstalled jet configuration by means of the nearfield Bruel \& Kjaer 1/4" 4935 microphone. The microphone was positioned in the target location of the plate trailing edge (Fig. 3). During the measurements of the installed jet, the near-field microphone was removed.

The position of the plate relative to the jet was chosen from the following considerations. As has been mentioned above, in most of the recent publications, ${ }^{5,6,9-11,13-17}$ jet-wing installation noise was studied for the case when the trailing edge lies in the linear hydrodynamic region of the jet near-field. In practice, such conditions may be realized for jetunder-the-wing configurations with not very large flap deflection angles. ${ }^{12}$ This allowed developing simple models, in which the field interacting with the plate trailing edge is modeled by the superposition of the near-field evanescent waves of different azimuthal numbers as if the plate is absent. ${ }^{6,10,11,13-17}$ In the current study, we are also focused on the configuration when the plate is not intruded into the jet shear layer so that linear mechanism of low-frequency installation noise is valid.

The distance between the plate and the jet axis $(h=0.8 D)$ was chosen to ensure reliable fixation of the plate. The plate was attached to the nozzle duct by a clamp. The longitudinal position of the trailing edge $(l=D)$ was determined by the two requirements. First, it should not be too small because installation noise decreases when the plate is shifted upstream. ${ }^{8}$ Second, as it was pointed out above, it should not be too large so as the trailing edge lied in the linear hydrodynamic region of the jet near-field.

\section{B. Results of the experiments}

At first stage of the experiment, adjustment of the excitation and control signals was performed. This was done for the isolated jet (the plate was not installed). A microphone was placed in the jet near field in the expected location of the plate trailing edge (Fig. 3). Typical near-field spectrum registered by the microphone is shown in Fig. 4a. The maximum of the spectrum corresponds to the hydrodynamic disturbances associated with shear layer instability waves. When the plate is inserted into the near-field of the jet, spectral properties of the pressure pulsations in the vicinity of the trailing edge define those for the far-field noise (see Eq. (1)). ${ }^{15}$

The jet was initially excited by the loudspeaker at $S t=0.6$ corresponding to spectral maximum. A peak at the excitation frequency appeared in the near field (Fig. 4a). In the signals registered by the arc microphones, the peak can also be observed, because of the transmission of the sound from inside the duct to the far-field. ${ }^{25,35}$

Then the jet was excited by the plasma actuator at the same frequency $S t=0.6$ (the loudspeaker was turned off). The amplitude of the excitation was adjusted in such a way as to align the peak value to that registered during the acoustic excitation. The spectra on the near-field microphone corresponding to the mutually adjusted acoustic and plasma excitations are shown in Fig. 4a. Note that in the far field the self-noise of the actuator was quite low - it is only $4 \mathrm{~dB}$ higher than the noise of the unexcited jet at $90^{\circ}$ in contrast to the loudspeaker, which generates noise $\sim 20 \mathrm{~dB}$ above the jet noise level.

After the adjustment of the amplitudes, the two actuators were turned on together, and the phase shift between them was varied. As the phase changed, the variation in the amplitude of the resulting peak in the near field was observed. Normalized (by the maximum value) amplitude of the peak (in linear scale) as a function of the phase shift between the two excitations is shown in Fig. 4b. The red curve in Fig. $4 \mathrm{~b}$ is the approximation by a simple function

$$
f(\Delta \varphi)=\frac{1+\delta \cos \left(\Delta \varphi+\varphi_{0}\right)}{1+\delta}
$$

where $\delta$ is the ratio of the peak amplitudes (taken in Pascals) of the two excitations (acoustic and plasma), $\varphi_{0}$ is the experimentally measured reference phase shift. Ideally, $\delta$ should be equal to 1 , but in real experiment it was estimated as 0.7 , because the peaks were not identical. 

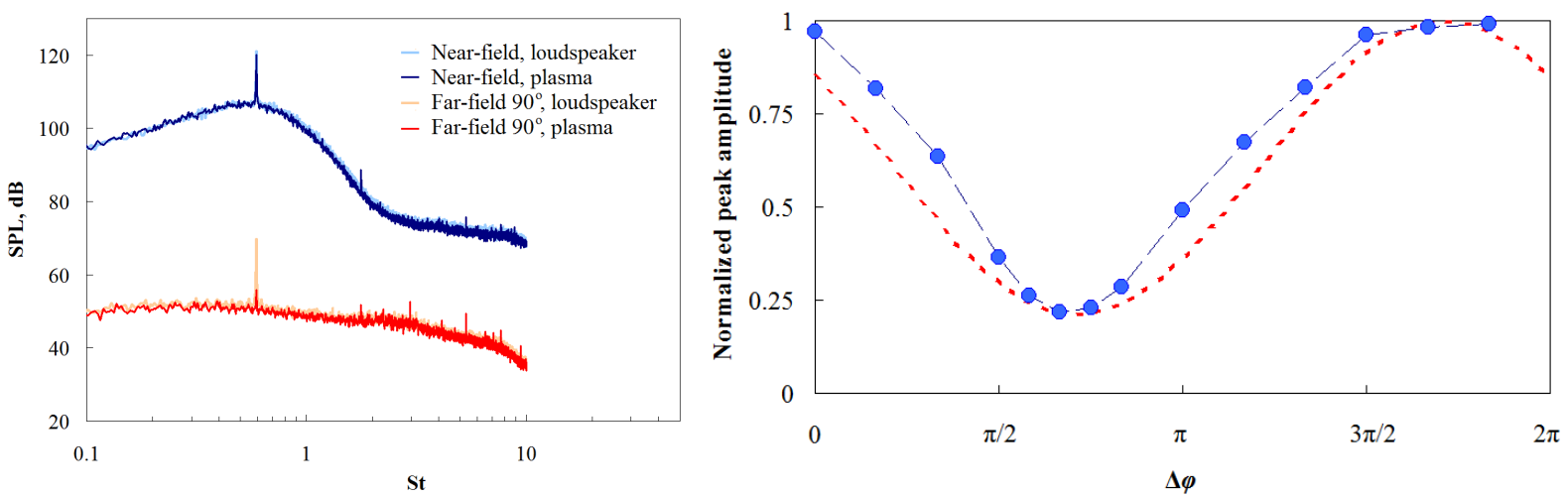

Figure 4. Control system adjustment. (a) - near-field and far-field $\left(90^{\circ}\right)$ spectra of the jet excited by the loudspeaker and HF DBD actuator (in different runs), the amplitudes of the peaks at the excitation frequency are tuned to be close to each other for the two excitations; (b) - evolution of the near-field peak as a function of the phase shift $\Delta \varphi$ between the loudspeaker and HF DBD actuator operating together, circles - experiment, red line - approximation (1).

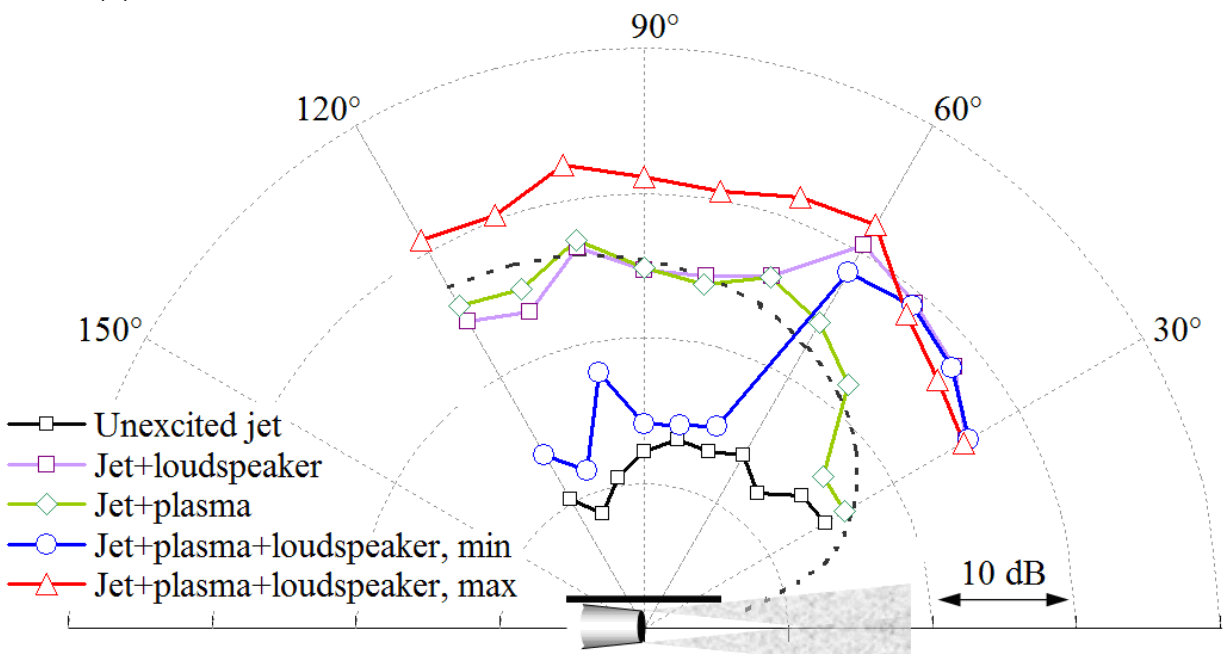

Figure 5. Far-field effect of the installation noise control for the excited jet. Black dotted line corresponds to a cardioid directivity $\left(\sim \sin ^{2}(\theta / 2)\right)$.

After the described procedure, we found the phase shifts corresponding to maximal amplification and attenuation of the resulting peak in the near field at the target location of the trailing edge. At the final stage of this experiment, the plate was installed near the nozzle. Unexcited jet noise was measured first, then acoustic and plasma excitation were activated in different runs, and finally both actuators were turned on simultaneously at different values of the phase shift $\Delta \varphi$.

The results are shown in Fig. 5, in which polar directivities of the acoustic field at the excitation frequency are plotted. It is seen that the excitation of the jet with one of the actuators (plasma or acoustics) leads to a significant intensification of the installation noise. This noise has a cardioid-like directivity in accordance with the analytical models. ${ }^{6,-11,13-16}$ Note also that the amplitudes of installation noise generated by acoustic and plasma actuators are very close to each other as expected from the near-field adjustment. It is also seen that when the phase shift corresponds to the maximal instability waves amplification, installation noise is also amplified by about 5-6 dB in full accordance with the initial assumptions. When the phase shift corresponds to the maximal instability waves attenuation, installation noise is attenuated by about $10 \mathrm{~dB}$ and becomes comparable to that of the unexcited jet. Note that noise attenuation is not observed at low observation angles $\left(30^{\circ}-60^{\circ}\right)$, where installation noise is small, because at these angles the signal is dominated by direct acoustic radiation out of the duct (from the loudspeaker) and is not affected by the plasma actuator that does not produce significant self-noise.

The results of the first experimental campaign demonstrate the validity of the basic assumptions of the of the installation noise control concept. 


\section{Control of installation noise of unexcited jet}

\section{A. Experimental setup}

After the confirmation of the basic points of the control strategy in the experiments with the excited jet, the second test campaign was prepared to test the concept on an unexcited jet. Again, a simplified model of the jet-wing configuration was considered. Experiments were carried out in TsAGI anechoic chamber AC-2. Round nozzle of diameter $D=50 \mathrm{~mm}$ were used together with the aluminum plate considered in Section III (Figs. 6, 7).

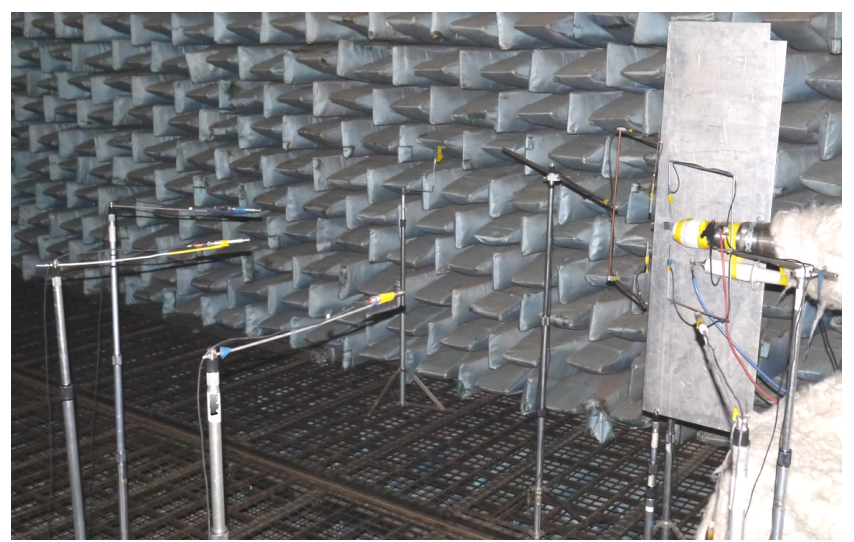

Figure 6. Jet-plate system in TsAGI anechoic chamber AC-2.

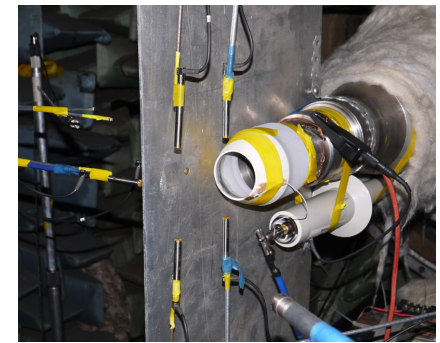

(a)

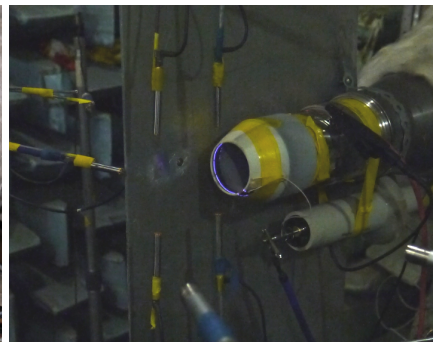

(b)

Figure 7. Control system testing: (a) - system is off; (b) - system is on.

We have used 11 microphones to control near- and far-field of the system: five microphones in the far-field (Bruel \& Kjaer, 1/2", type 4189, denoted hereafter as F1-F5) and six microphones in the near-field (Bruel \& Kjaer, 1/4", type 4935, denoted hereafter as N1-N6). Sketch of the near- and far-field microphones arrangement is shown in Fig. 8. Far-field microphones were located on the arc of radius $1.2 \mathrm{~m}$, which was centered on the plate trailing edge. Two of the microphones (F1 and F2) were located in the points symmetric relative to the plate at an angle $120^{\circ}$ to the flow direction in order to ensure that the spectra on both sides of the plate are similar at low frequencies (this should be the case for the installed configuration). ${ }^{6,8,11,17}$ In the near field, four microphones (N1-N4) were installed in the jet symmetry plane parallel to the plate at two jet cross-sections, the remaining two microphones were installed in the plate plane - the first (N5) was flush mounted in the plate, the second (N6) was installed just after the plate trailing edge. The coordinates (non-dimensionalized by the nozzle diameter) of the near-field microphones in the reference frame shown in Fig. 8 were as follows: $\mathrm{N} 1-(0.4,0.0,0.8), \mathrm{N} 2-(0.4,0.0,-0.8), \mathrm{N} 3-(1.7,0.0,0.95), \mathrm{N} 4-(1.7,0.0,-0.95), \mathrm{N} 5-$ $(1.0,-0.95,0.0), \mathrm{N} 6-(1.7,-0.95,0.0)$. The trailing edge was characterized by the $(x / D, y / D)$ pair $(1.6,-0.95)$.

Auxiliary measurements of the uninstalled jet were also performed (without the control). In this case, the microphone N5 was located symmetrically to the microphone N6 in the point $(1.7,0.95,0.0)$. 


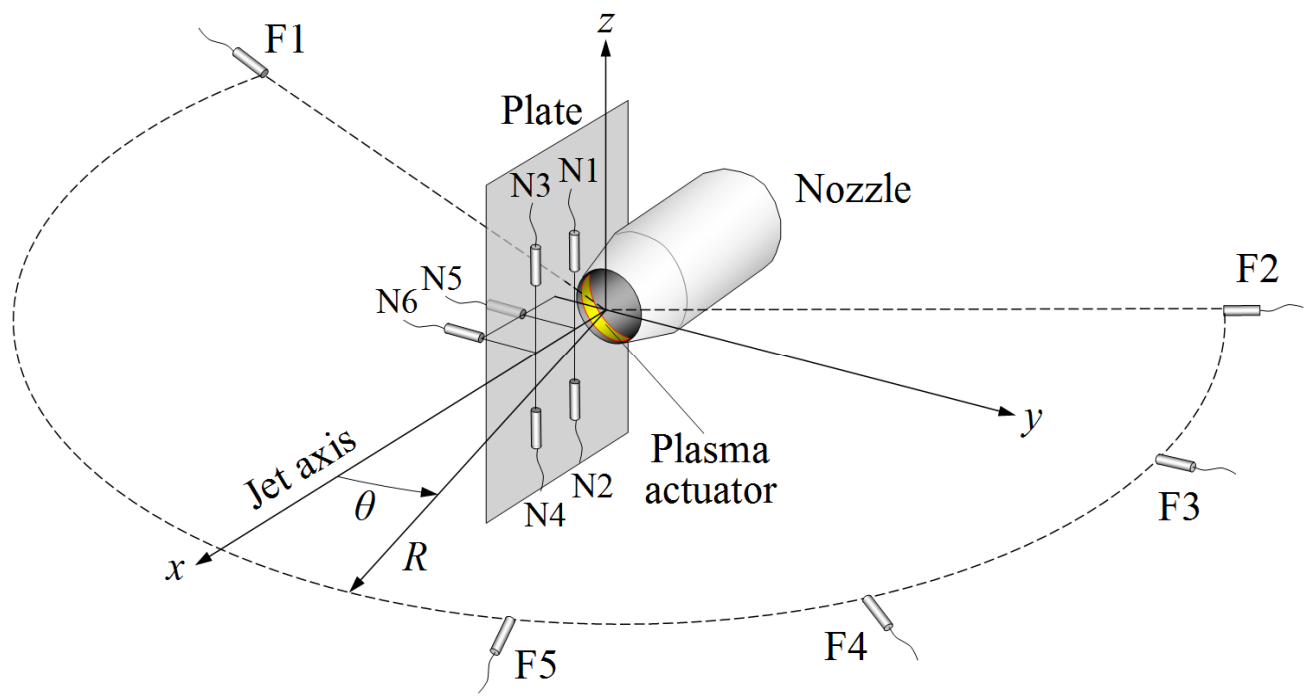

Figure 8. Sketch of the near- and far-field microphones location.

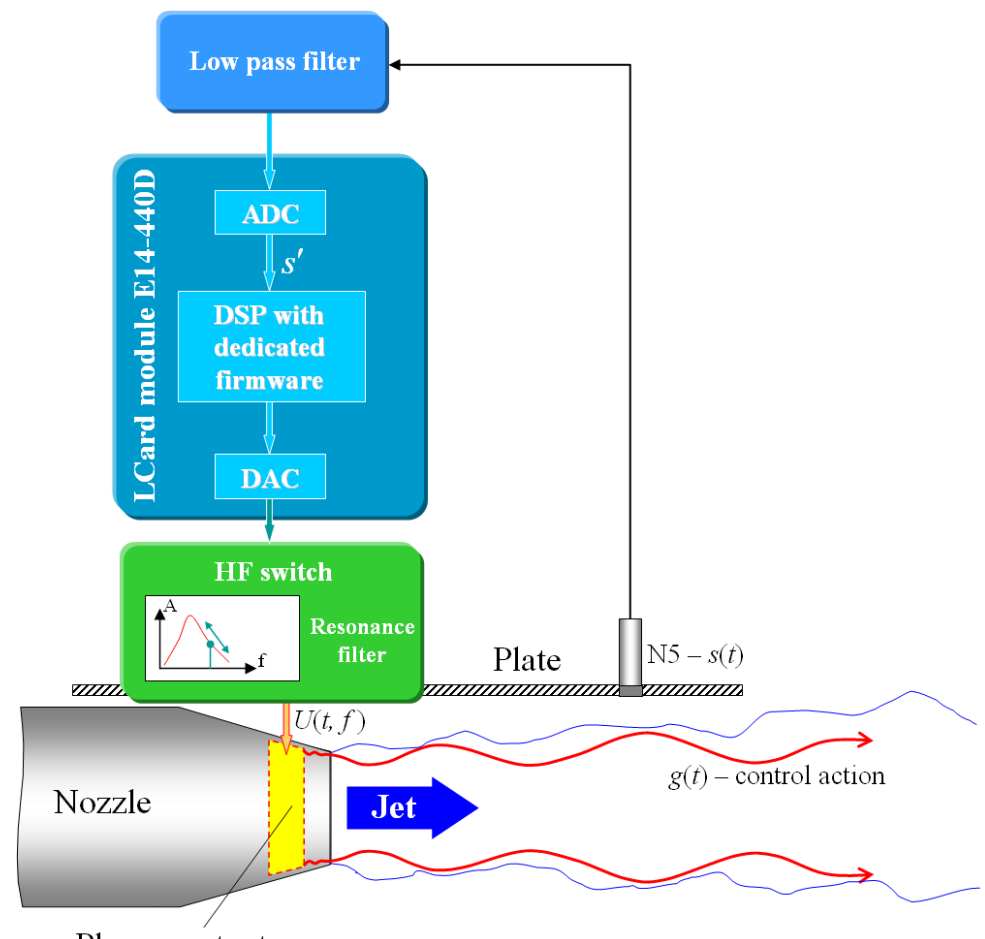

Plasma actuator

Figure 9. Schematic diagram of the closed-loop control system for the unexcited installed jet.

In order to generate instability waves, HF DBD actuator mounted on the inner surface of the nozzle was used in the same way as it is described in Section III and in the Part I of the study. ${ }^{1}$ Metal nozzle was covered by a ceramic coating $\sim 1 \mathrm{~mm}$ thick. Discharge was initiated on the edges of the ring metallic strip, glued to the internal nozzle surface at a distance 3-5 $\mathrm{mm}$ from the nozzle edge. The nozzle itself served as the second electrode. Highfrequency voltage was supplied by the transistor switch with a resonant transformer used as output. Actuator was powered by the sine voltage with amplitude $5-10 \mathrm{kV}$ at frequency $\sim 180 \mathrm{kHz}$.

The control system was identical to that used by Bychkov et al. ${ }^{1}$ for free jets, and the reader is referred to that work for more detailed description of the system. It was implemented on the basis of the LCARD module E14440D, integrating an ADC, DAC and a digital signal processor (DSP) with a clock frequency of $48 \mathrm{MHz}$. The 
capabilities of the module allow polling up to 16 microphones with sampling frequency of $25 \mathrm{kHz}$. In the current experiment, the signal from one microphone was polled up with $100 \mathrm{kHz}$ frequency. The signal is processed in real time by the DSP. The code of the module driver was modified to include digital filtering of the signal from the microphones, correction of phase and amplitude distortions, selection of a spatial mode by linearly converting the signal from several microphones. The processed signal is fed to the output through the DAC and further used for modulating the voltage at the discharge on the plasma actuator. The schematic diagram of the system is shown in Fig. 9. To form a control action in the jet-plate configuration, we used one sensor on the plate: flush mounted microphone $\mathrm{N} 5$ or trailing edge microphone N6.

The signal from the microphone was postprocessed by FIR filter was built into the code of the hardware:

$$
Y_{n}=\sum_{k=0}^{N-1} b_{k} X_{n-k}
$$

where filter coefficients are taken as $b_{k}=\frac{2}{N} K(k) \cos \left(\frac{2 \pi k i}{N}\right), i=0 \ldots N-1, K(k)$ is the window function. For the current study, we used rectangular window $K(k)=1$ and $N=128$ samples. Application of this filter is equivalent to the calculation of the coefficient of the window Fourier transform at the cosine of the $k$-th harmonic for a signal of $N$ samples. The applied transformation can be characterized by means of amplitude-frequency and phase-frequency characteristics (frequency response and phase response, respectively) as discussed in Bychkov et al. ${ }^{1}$

\section{B. Results of the experiments}

The adjustment of the system parameters was performed manually. At first step, the parameters of the FIR filter were chosen (central frequency $f_{c}$, number of samples $N$, window function) and the modified firmware is loaded into LCARD module. Then, modulation factor $\beta$ (i.e. transmission coefficient between the filtered signal from the reference signal and the voltage applied to the actuator) and time delay $\tau$ (it is almost equivalent to phase shift for narrowband signals) were set in order to obtain the desired control effect. It is obvious that for effective attenuation of NIW, and hence installation noise in the far field, the control instability wave should have the same amplitude as NIW and be in antiphase to them. The procedure of the system adjustment in the $(\tau, \beta)$ space is sketched in Fig. 10. Each point on the plot denotes one measurement with the activated control system with corresponding values of $\tau$ and $\beta$. After the first measurement (initial guess), the step-by-step correction of the $(\tau, \beta)$-pair is performed, with only one parameter being changed at one step. The target effect is the maximum attenuation of the signal around the central frequency of the FIR filter. When such "optimal" pair is found, several measurements with different time delays are performed (within one typical period $\Delta \tau \approx 1 / f_{c}$ of the narrowband signal) in order to investigate not only the NIW suppression effect but NIW intensification as well. In the current paper, we shall present the results for maximum attenuation of noise.

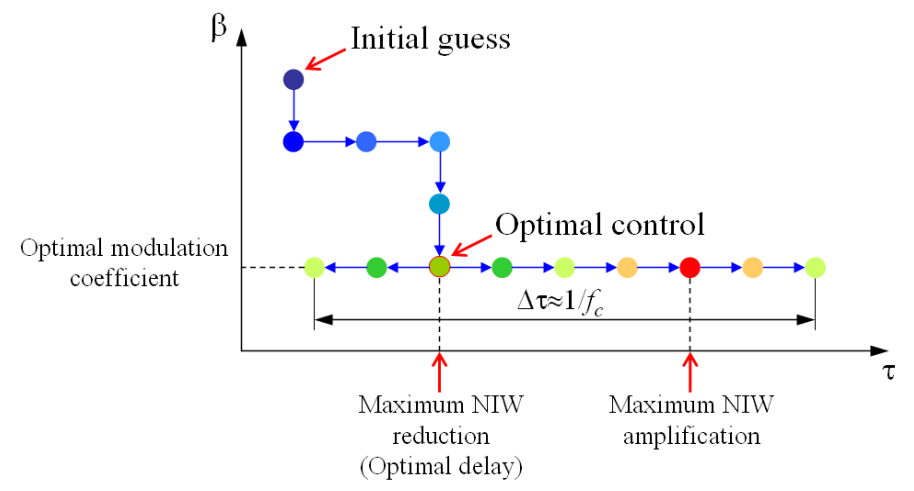

Figure 10. Sketch of the control system adjustment procedure.

Let us first consider the characteristics of the uncontrolled jet. In Fig. 11a, the near-field pressure spectra are shown. The spectra have typical shape with strong low-frequency hump associated with the hydrodynamic near-field 
fluctuations of the jet and high-frequency part with inverse square decay corresponding to the acoustic perturbations. Detailed analysis of the near-field spectra structure may be found in the work of Arndt et al. ${ }^{36}$

In addition to the spectra of the total signals, we also decomposed the signals measured by the pairs N1-N2 and N3-N4 into symmetric and antisymmetric components. It was previously shown ${ }^{31,32,34}$ that the first two azimuthal modes dominate in the irrotational near field of the jet, therefore a pair of symmetrically located microphones allows extracting principal information on the modal content in accordance with the simple formulas

$$
\begin{aligned}
& a_{0}(x, t)=\left(s_{1}(x, t)+s_{2}(x, t)\right) / 2, \\
& a_{1}(x, t)=\left(s_{1}(x, t)-s_{2}(x, t)\right) / 2,
\end{aligned}
$$

where $s_{1}$ and $s_{2}$ are the signals measured in the symmetric opposite points in the jet near-field at some cross-section $x$. For the pair N1-N2 we will denote symmetric and antisymmetric components as $a_{0}\left(x_{1}\right)$ and $a_{1}\left(x_{1}\right)$, and for the pair N3-N4 as $a_{0}\left(x_{2}\right)$ and $a_{1}\left(x_{2}\right)$, respectively.

For the case of the uninstalled jet, four microphones N3-N6 located in one cross section allowed to perform more accurate decomposition into azimuthal components and to resolve the first three modes

$$
\begin{aligned}
& a_{0}(x, t)=\left(s_{1}(x, t)+s_{2}(x, t)+s_{3}(x, t)+s_{4}(x, t)\right) / 4, \\
& a_{1}(x, t)=\left(s_{1}(x, t)-s_{3}(x, t)\right) / 2, \\
& b_{1}(x, t)=\left(s_{2}(x, t)-s_{4}(x, t)\right) / 2, \\
& a_{2}(x, t)=\left(s_{1}(x, t)-s_{2}(x, t)+s_{3}(x, t)-s_{4}(x, t)\right) / 4,
\end{aligned}
$$

where $a_{i}$ and $b_{i}$ are the modal coefficients in the decomposition

$$
s_{i}(x, t) \approx a_{0}(x, t)+a_{1}(x, t) \cos \theta_{i}+b_{1}(x, t) \cos \theta_{i}+a_{2}(x, t) \cos 2 \theta_{i}+\ldots,
$$

$\theta_{i}$ is the azimuthal angle corresponding to the location of the $i$-th microphone.

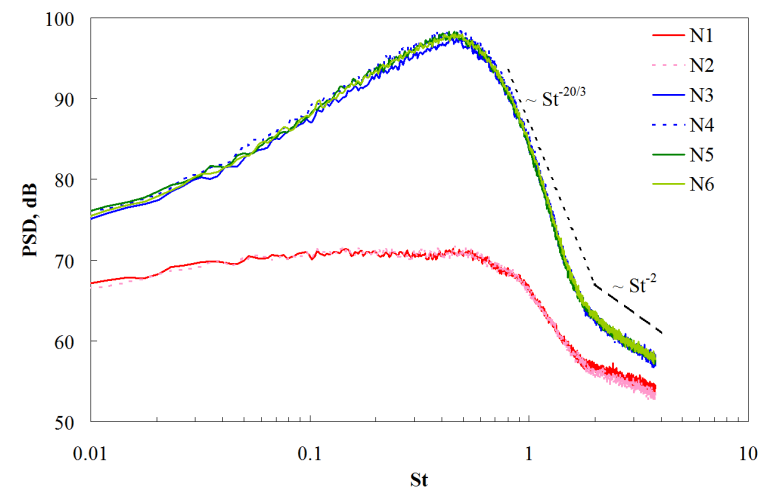

(a)

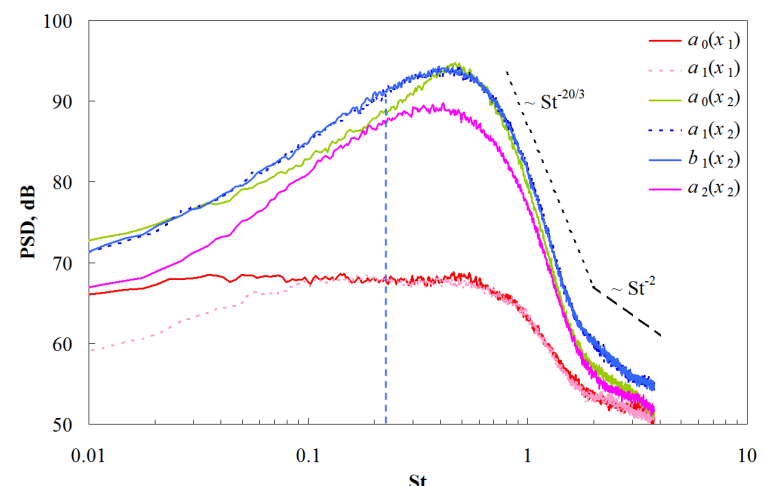

(b)

Figure 11. Near-field spectra of the uninstalled jet. (a) - total spectra; (b) - modal spectra.

Modal components of the near pressure field of the uninstalled jet, calculated by Eq. (3), are plotted in Fig. 11b. For convenience, the target frequency for control is also shown by a vertical dashed line. It can be seen that in the first cross-section (microphones N1, N2) the symmetric and antisymmetric modes spectra are quite close to each other, however, one should keep in mind that two microphones may not be enough to separate all the modes. Indeed, for the next cross-section (microphones N3-N6), it is seen that for $0.4<S t<1$ the first two modes dominate the signal and are almost equal to each other. However, for lower frequencies $(0.07<S t<0.3)$, the first mode starts to dominate, while the axisymmetric mode $a_{0}$ becomes relatively small and close to the second mode $a_{2}$. Note that two-microphone decomposition (2) can not distinguish between $a_{0}$ and $a_{2}$ (see Eq. (4)) if they have comparable amplitudes. Let us mind this effect for future analysis.

Figure 12 presents the same characteristics for the installed jet. Interestingly, that installation of the plate leads to some changes in the modal content of the near field, namely, the first pair of microphones demonstrates that the 
axisymmetric mode begins to dominate the signal in the presence of the plate. This effect is not quite clear up to now and requires further, more careful, investigation.

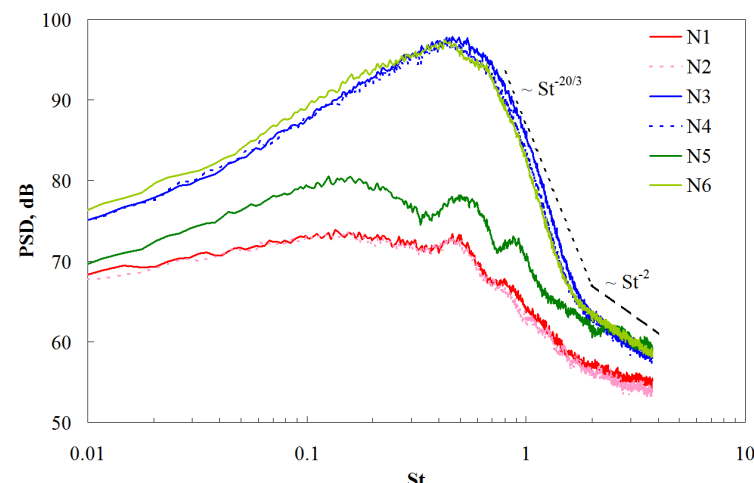

(a)

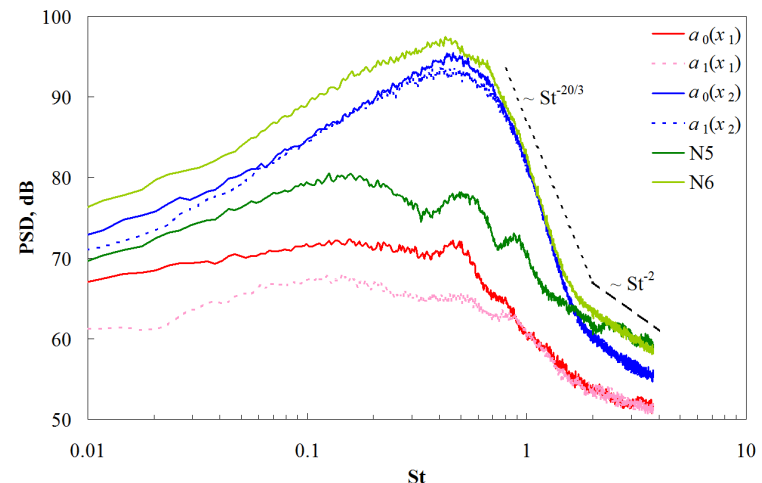

(b)

Figure 12. Near-field spectra of the installed jet. (a) - total spectra; (b) - symmetric $\left(a_{0}\right)$ and antisymmetric $\left(a_{1}\right)$ components. Control is off.

Comparison of the acoustic spectra in the far field for the uninstalled and installed jets are presented in Figs. 13, 14. One can see typical low-frequency $(S t<0.8)$ installation noise. This noise is symmetric relative to the plate and more pronounced in upstream and sideline directions of observation due to cardioid-like directivity. For clarity, in Fig. 14, the two sets of spectra are superimposed and the low-frequency installation effect caused by the trailing edge scattering of instability waves is highlighted by grey color.

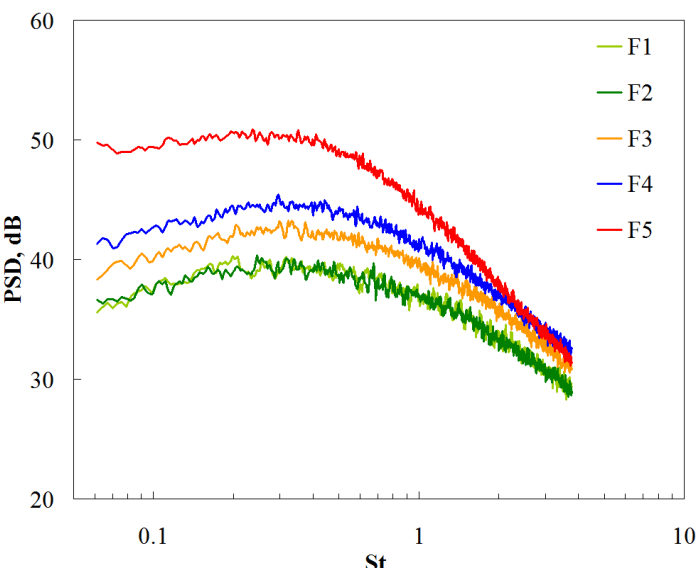

(a)

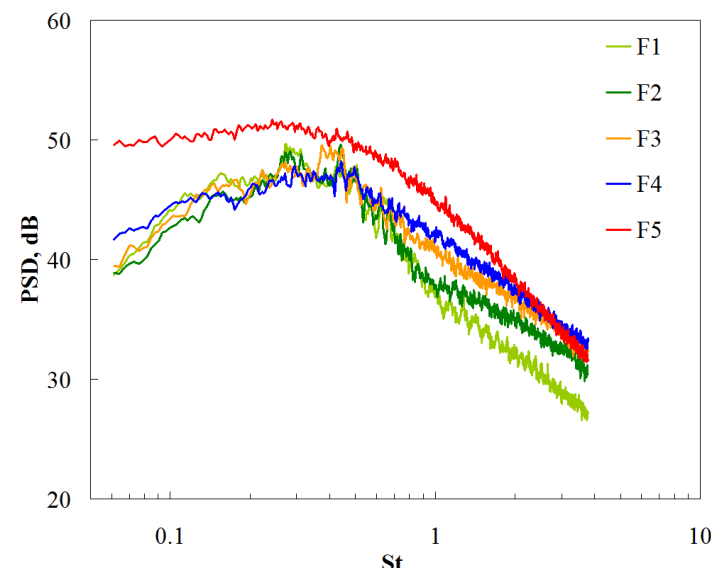

(b)

Figure 13. Far-field acoustic spectra: (a) - uninstalled jet; (b) - installed jet. Control is off.

Figure 15 demonstrates the spectra of the symmetric and antisymmetric noise components in the far field calculated by Eq. (2) and the signals from the microphones F1 and F2. It is clearly seen that low frequency installation noise has antisymmetric behavior in the far field reflecting its dipole nature. ${ }^{17}$ In view of further investigation of the effect of control on the far-field spectra we also estimated the confidence interval related to nonperfect repeatability of jet operating conditions. Figure 16 shows spectra for the microphone F2 for eight runs of one and the same jet in the course of the test campaign. It can be seen that, at low frequencies of interest, all of the spectra lies in $0.5 \mathrm{~dB}$ interval.

One can also see that the part of the spectra on the microphones F1, F2 in the Strouhal number range 0.2-0.25 is quite smooth and free of peaks. Therefore, this range is more convenient for the observation of control effect. As expected, it should be more pronounced for the microphones F1, F2 where installation noise is strong enough. It was one of the reasons for choosing the central frequency of the FIR filter for the control corresponding to Strouhal number $S t=0.23$ 


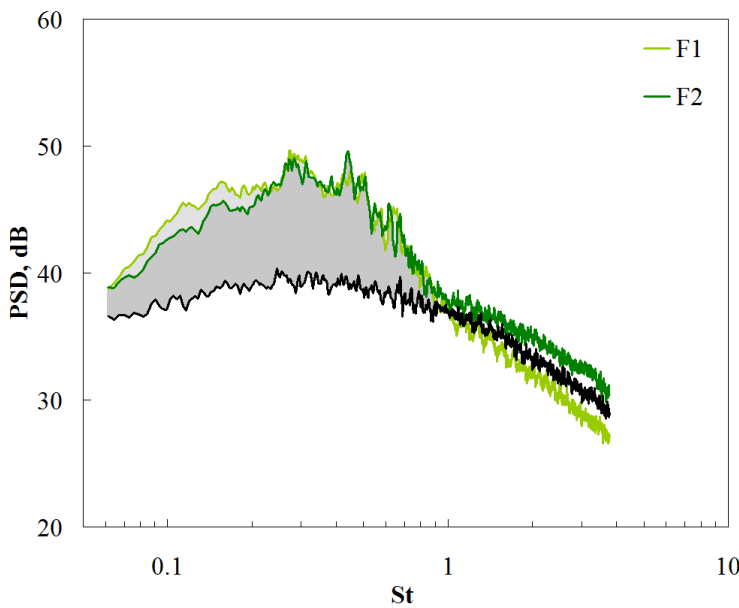

(a)

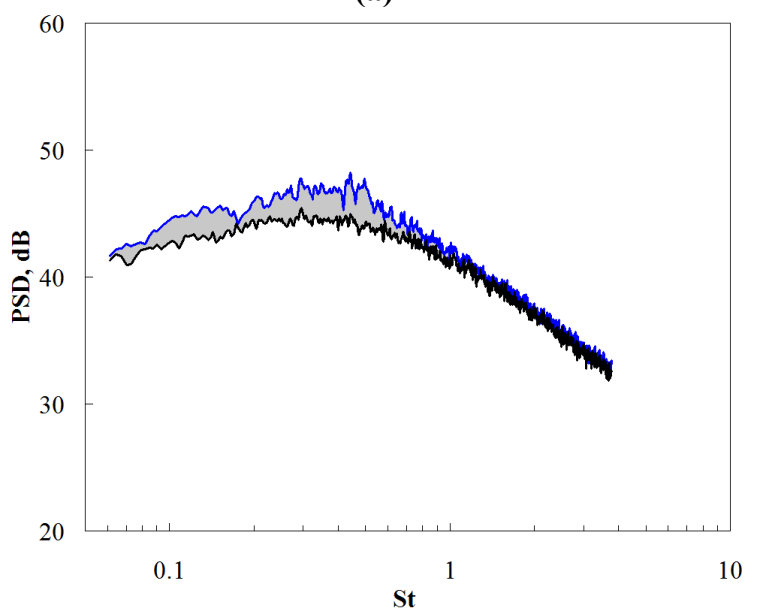

(c)

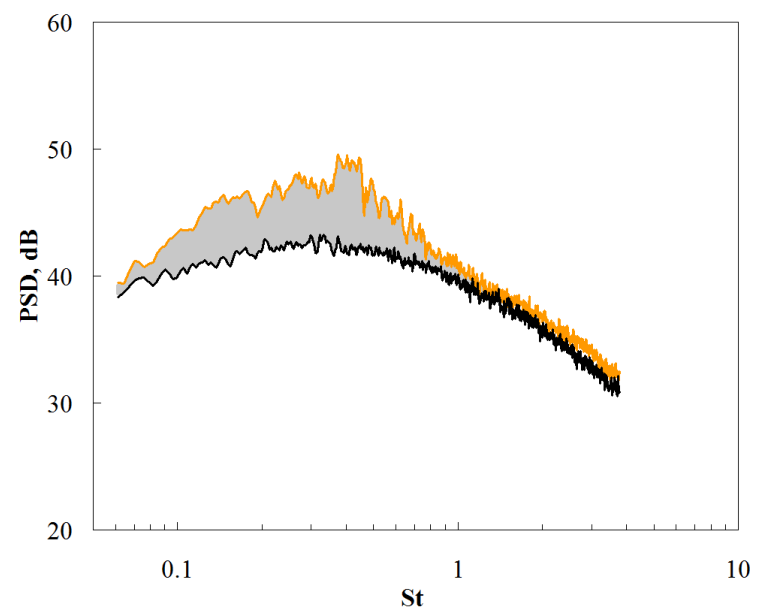

(b)

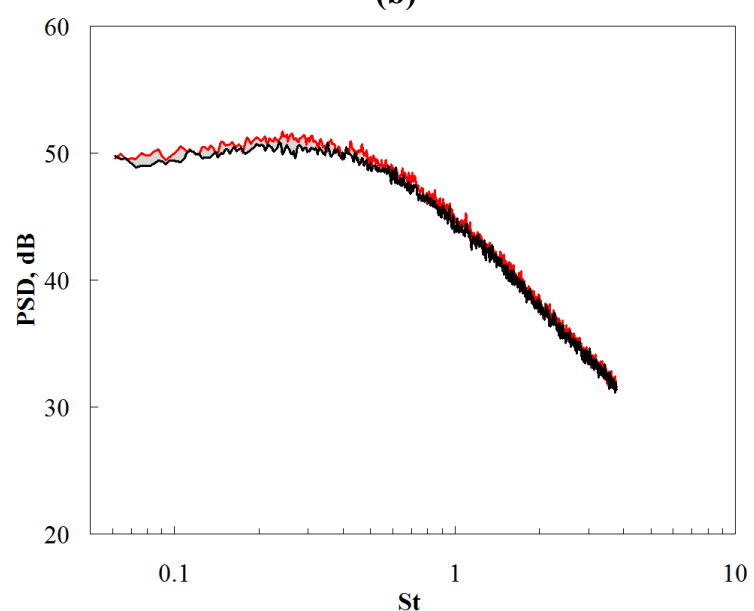

(d)

Figure 14. Jet installation noise. Comparison of the spectra of the free and installed jets. (a) - microphones F1, F2; (b) - microphone F3; (c) - F4; (d) - F5. Black curves correspond to the noise of the free jet. Grey zones indicate low-frequency installation effect caused by the trailing edge scattering of instability waves. Control is off.

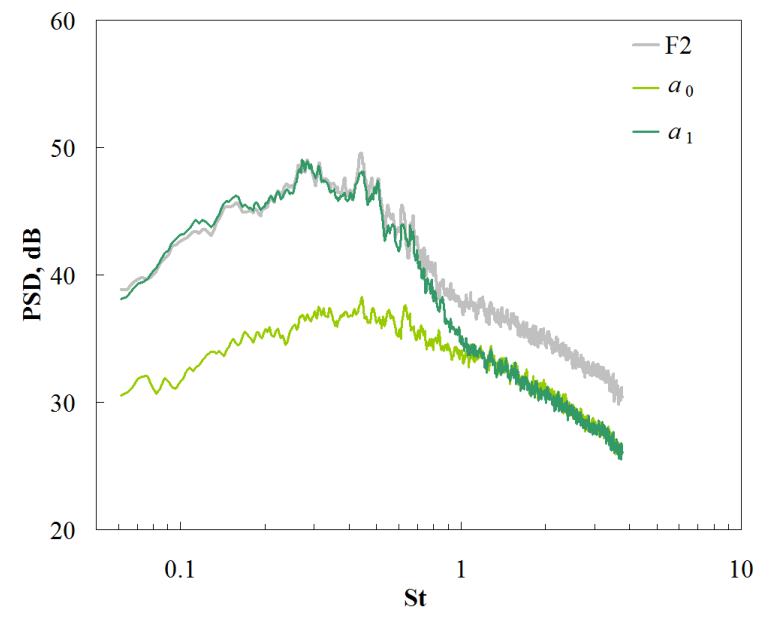

Figure 15. Decomposition of the installation noise into symmetric $\left(a_{0}\right)$ and antisymmetric $\left(a_{1}\right)$ components. 


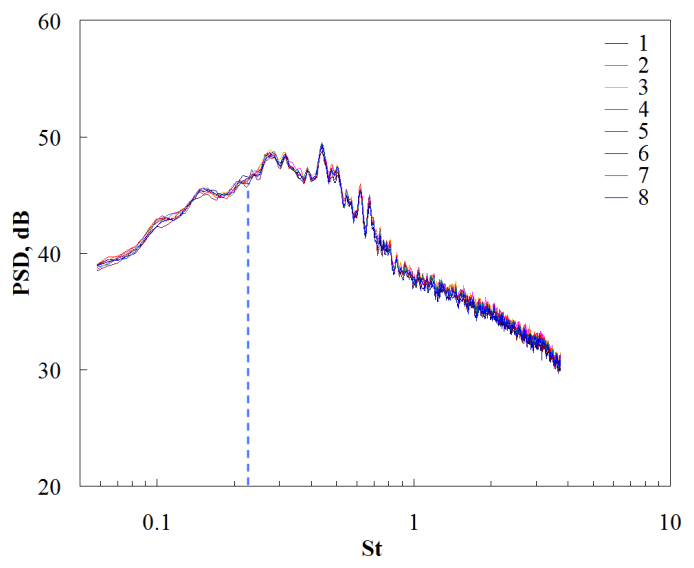

Figure 16. Estimation of the confidence interval due to nonperfect repeatability of jet operating conditions. Spectra for the microphone F2 are shown for eight runs. Control is off. For convenience, the control frequency is marked by a blue dashed line.

Now let us consider the results for the controlled jet. The results reported below were obtained with the reference microphone N5. In Fig. 17a, the near-field pressure spectra are shown. It can be clearly seen that the effect of control is only noticeable for the reference microphone N5 and the pair N1-N2. At the central frequency, reduction of the signal by about $2.5 \mathrm{~dB}$ is observed.

It is interesting to look at the symmetric and antisymmetric components of the controlled jet. Corresponding spectra are shown in Fig. 17b. These components are calculated in accordance with Eq. (2). It can be seen that the effect of control is localized in the symmetric mode only for the microphone pair N1-N2. This is quite natural result because we used axisymmetric ring-like electrode in the actuator. Thus, the actuator indeed generated axisymmetric control disturbances. However, the control action is not observed at the axisymmetric mode spectrum corresponding to the pair N3-N4, and at the signal N6. This can be related to the fact that these microphones were located farther downstream from the reference microphone N5, and they were closer to the jet boundary so that other, not resolved, modes could contribute to the signal. Thus, one pair of microphones in the downstream section may not be enough to extract axisymmetric mode from the total signal. This effect was observed in case of the uninstalled jet, where the axisymmetric and second modes were of comparable amplitudes in this cross-section. Another factor that could have influence on the modal content in the location of microphones N3, N4, N6 may be related to the presence of the plate, so that the field structure may become asymmetric on average. In this case, the azimuthal decomposition of such a field may become uninformative. In any case, the visual absence of the control effect on the spectra in the downstream section indicates that the influence of the axisymmetric control is small on the background of other disturbances.

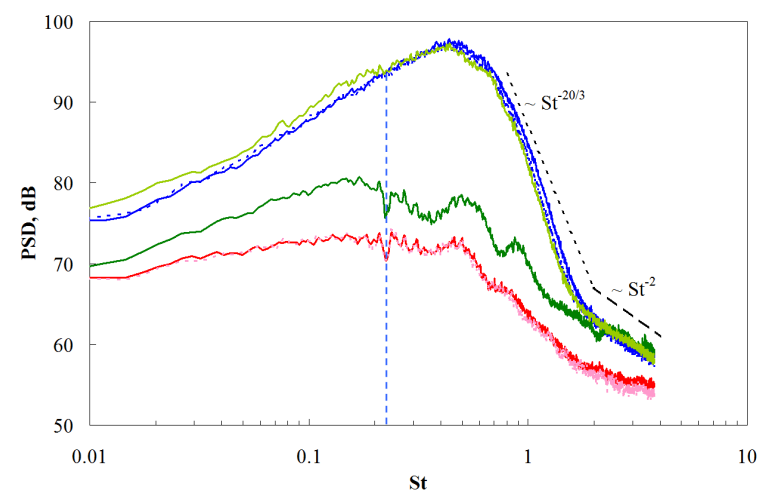

(a)

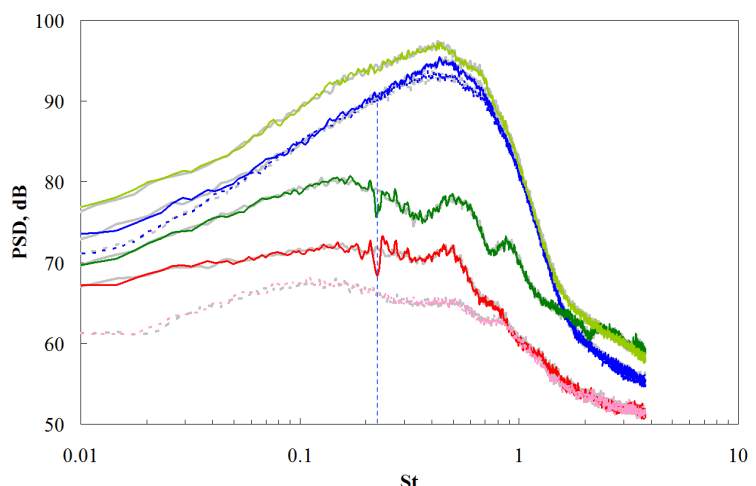

(b)

Figure 17. Near-field spectra of the installed jet. (a) - total spectra; (b) - symmetric $\left(a_{0}\right)$ and antisymmetric $\left(a_{1}\right)$ components. Control is on. Grey curves correspond to the reference spectra of the uncontrolled jet. See Fig. 12 for notation. The control frequency is marked by a blue dashed line. 


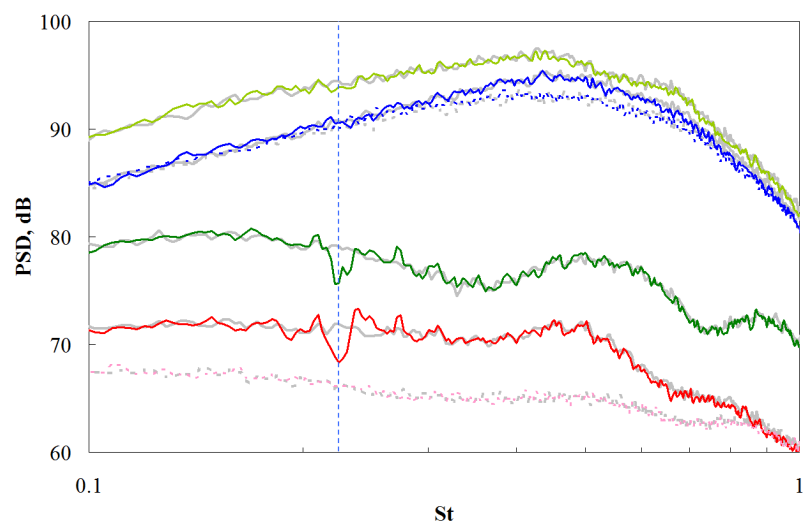

Figure 18. Close-up view on the Fig. 17b around the control frequency. See Fig. 12b for notation. Grey curves correspond to the reference spectra of the uncontrolled jet.

Figure 18 demonstrates the close-up view of the near-filed spectra around the control frequency. It is also seen that the attenuation of fluctuations in the frequency band defined by the FIR filter setting is accompanied by their amplification on both sides of the central frequency. This effect is related to the phase properties of the filter and is discussed in detail in Part I of the study. ${ }^{1}$

Additional information on the influence of the control on the measured signals can be obtained from the coherence functions analysis. Coherence between the reference microphone N5 and TE microphone N6, and symmetric and antisymmetric components of the near field measured by the microphone pairs N1-N2 and N3-N4 is plotted in Fig. 19 for the uncontrolled and controlled jets. It is seen that the control leads to the drop in the coherence for the symmetric modes and for the signal N6. These results show that the effect of control is noticeable not only for the microphones N1, N2 (as seen from the spectra), but for downstream microphones N3, N4, N6 as well, although it is not visible on the spectra. Therefore, the control indeed leads to the reduction of the axisymmetric mode in the vicinity of the trailing edge.

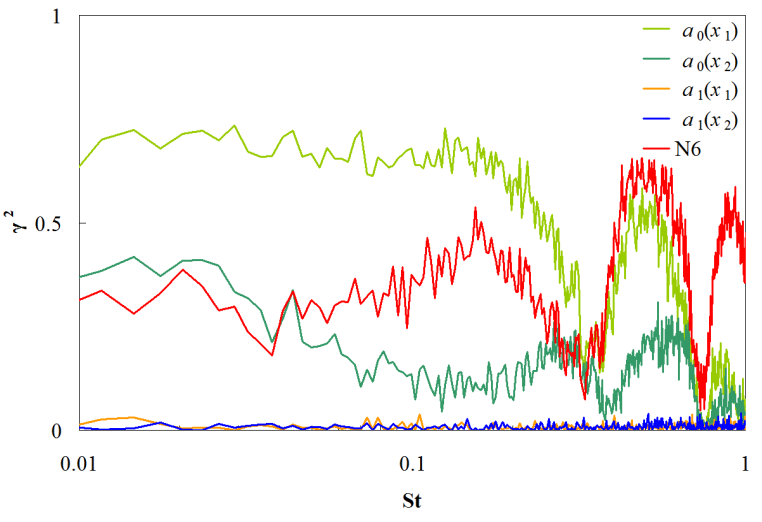

(a)

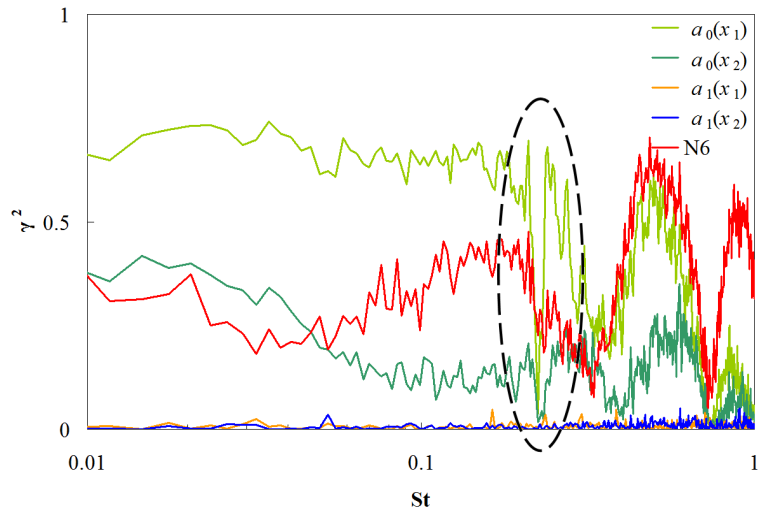

(b)

Figure 21. Coherence between the reference microphone N5 and: TE microphone N6, symmetric $\left(a_{0}\right)$ and antisymmetric $\left(a_{1}\right)$ components of the near field measured by the microphone pairs N1-N2 and N3-N4 in the cross sections $x_{1}$ and $x_{2}$, respectively. (a) - control is off; (b) - control is on. 


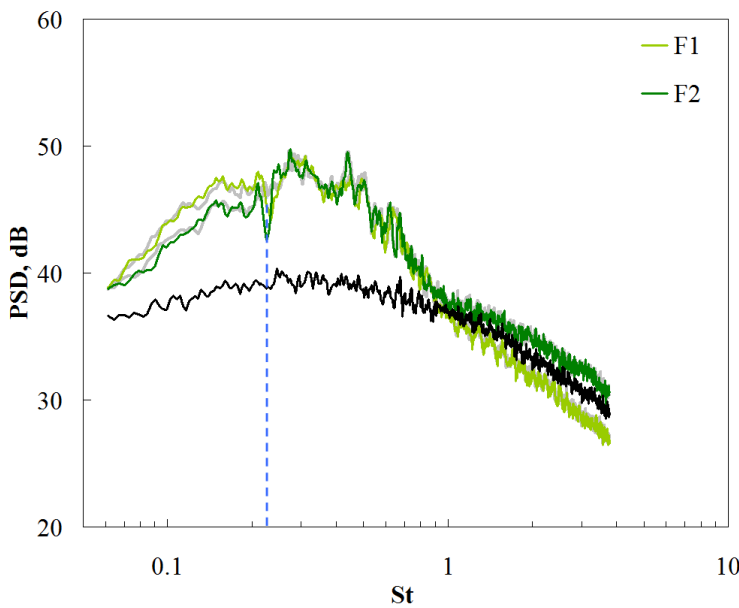

(a)

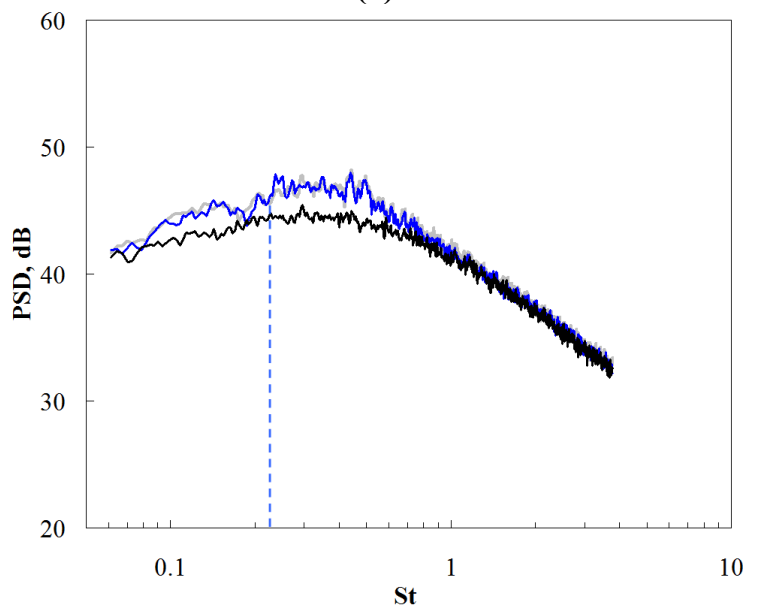

(c)

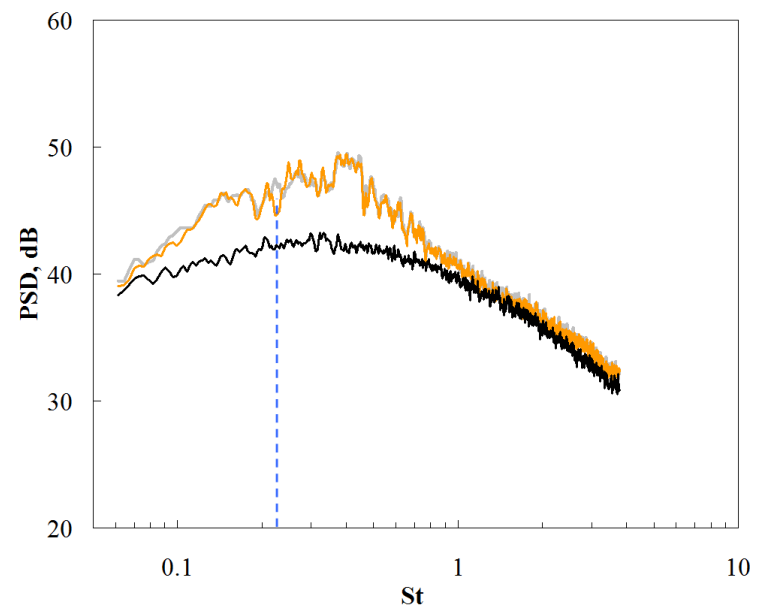

(b)

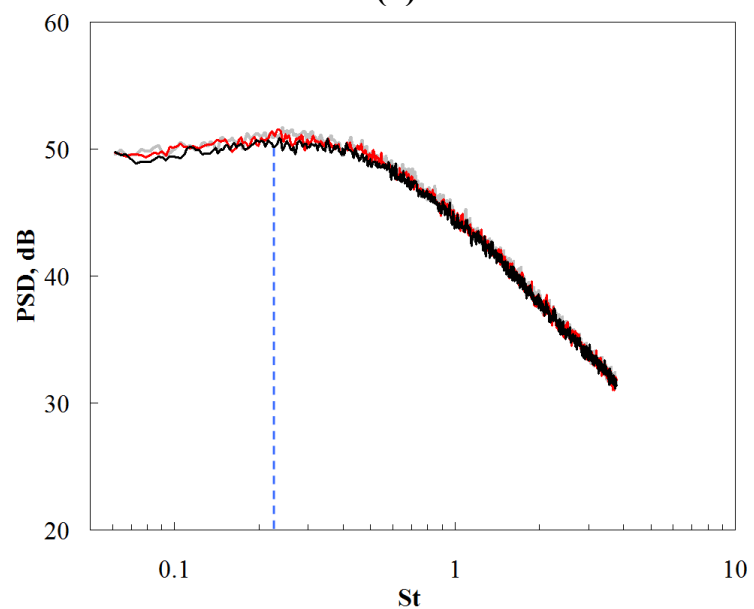

(d)

Figure 19. Jet installation noise. Comparison of the spectra for the free and installed jets. (a) - microphones F1, F2; (b) - microphone F3; (c) - F4; (d) - F5. Control is on. The control frequency is marked by a dashed line. Black curves correspond to the noise of the free jet, grey curves correspond to the noise of the uncontrolled installed jet.

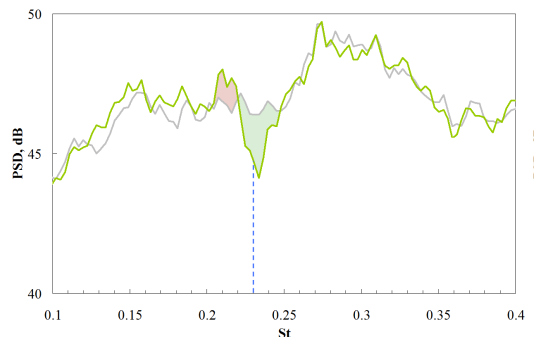

(a)

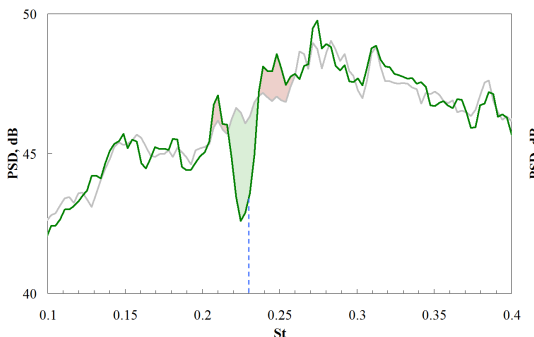

(b)

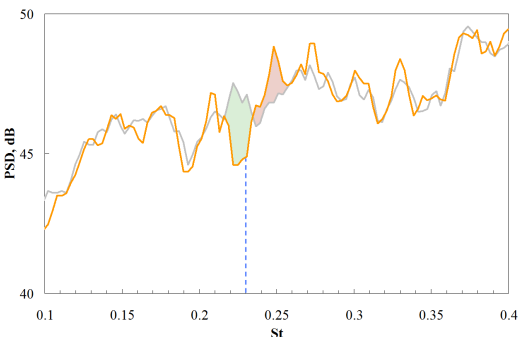

(c)

Figure 20. Close-up view on the spectra of the controlled jet. (a) - microphone F1; (b) - F2; (c) - F3. Grey curves correspond to the noise of the uncontrolled installed jet. Zones of noise reduction and amplification are highlighted by green and light red, respectively. 


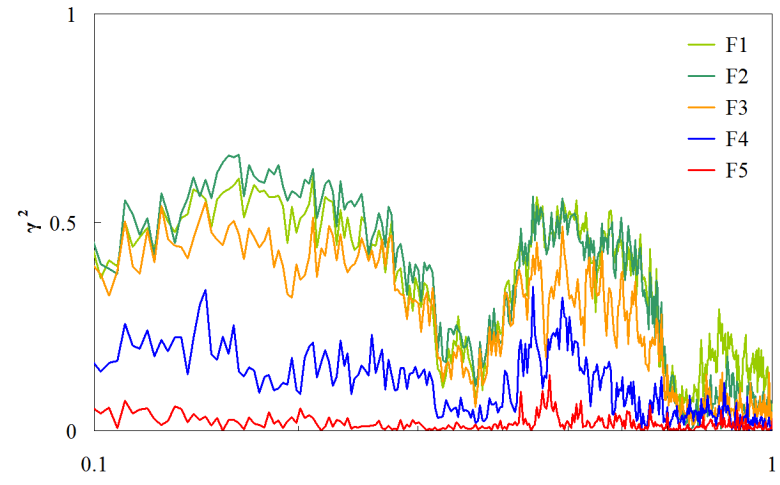

(a)

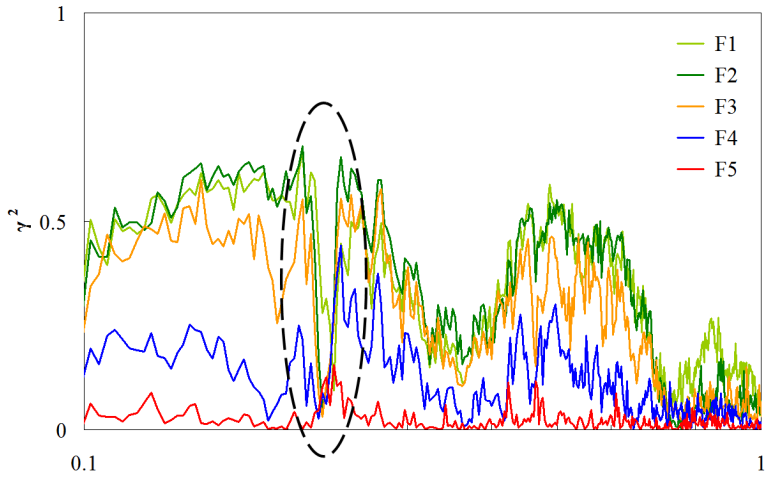

St
(b)

Figure 21. Coherence between the reference microphone N5 and far-field microphones F1-F5. (a) - control is off; (b) - control is on.

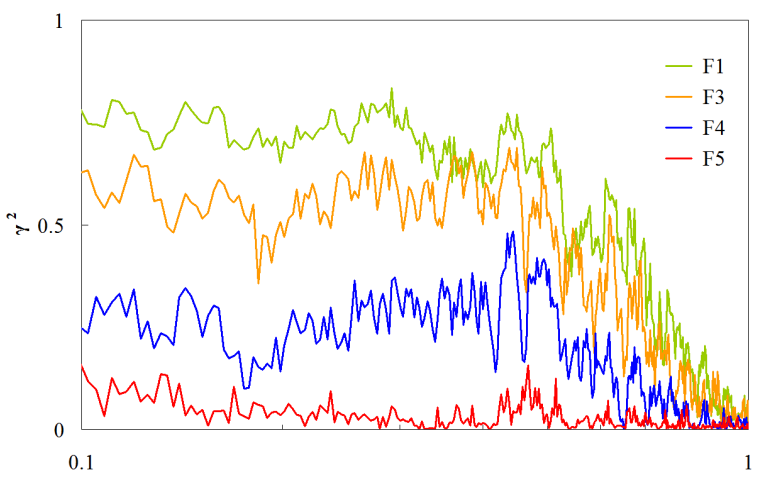

(a)

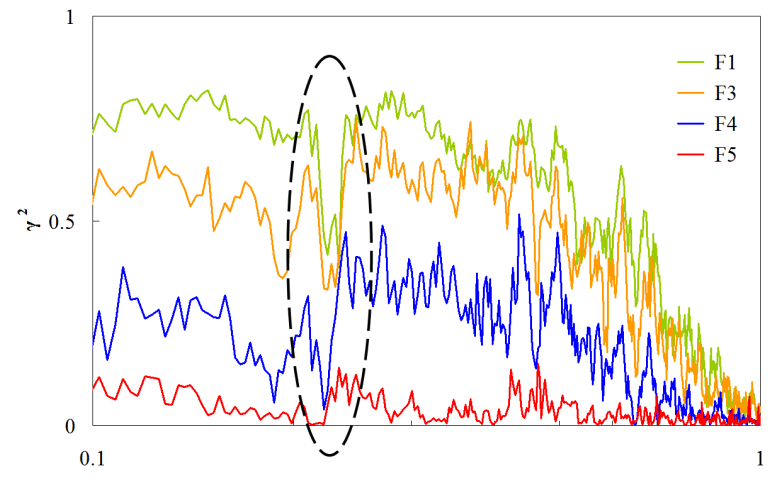

(b)

Figure 22. Coherence between the microphone F2 and far-field microphones F1, F3, F4, F5. (a) - control is off; (b) - control is on.

Consider the results obtained for the far field. Corresponding spectra for the controlled jet are shown in Fig. 19 along with the reference spectra of free jet noise (black curves) and uncontrolled installed jet noise (grey curves). The effect of control is clearly seen for the upstream (microphones F1, F2) and sideline (F3) directions. Farther downstream (microphones F4, F5), the effect is not visible on the noise spectra. For the microphones F1, F2, and F3, the close-up view on the spectra of the controlled jet is shown in Fig. 20. It is seen that the effect of noise reduction at the target frequency reaches $2.5 \mathrm{~dB}$ for the upstream microphones that is well beyond the confidence interval related to the repeatability of the operating conditions. One can notice that the zones of noise reduction (highlighted by green in Fig. 20) are accompanied by the zones of noise amplification (highlighted by light red in Fig. 20). This effect has the same origin as for the near field. It is caused by the phase properties of the FIR filter. Time delay, which is inherent to the feedback control system, leads to the nonzero slope of the phase characteristic of the filter. Therefore, amplification zones appear on the sides of the main frequency when the phase shift reaches $\pi$ (relative to the central frequency). This effect is discussed in more detail in Bychkov et al. ${ }^{1}$

The absence of the control effect on the microphones F4 and F5 may be explained by the fact that the contribution of the installation noise into the total signal decreases for downstream observation angles due to specific directivity of the former. However, analysis of the coherence functions shows that the control effect is still detected at the downstream microphone F4. Coherence functions between the reference microphone N5 and the farfield signals are shown in Fig. 21 for the uncontrolled and controlled jets. Significant values of the coherence for the microphones F1-F4 displays the fact that installation noise contribute to the total signal. It is seen that the control initiation leads to a significant drop in the coherence for the microphones F1-F4. The same effect is illustrated in Fig. 22 using the coherence functions between the far-field microphone F2 (which is dominated by the installation noise), taken as the reference one, and the remaining far-filed microphones. Again, one can see that installation 
noise is pronounced at all of the microphones except F5, which is strongly dominated by the jet noise. Therefore, the effect of control can be observed as a coherence drop at the target frequency for the signals F1-F4.

Note finally that the case with the reference microphone located at the trailing edge (N6) was also investigated. However, the effect of control was somewhat lower than that for the microphone N5. This may be due to the fact that the signal on the microphone N6 is dominated by the first azimuthal mode for the Strouhal number corresponding to the central frequency of the FIR filter (see Fig. 11b). Therefore, the location of the microphone N6 is not optimal for the realization of the control of the axisymmetric disturbances, and vice versa, the location of the microphone N5 suits quite well for the system operation because its signal mainly consists of the axisymmetric fluctuations. This reflects the importance of choosing the right place for the reference signal.

\section{Conclusions}

In the current paper, which is the Part II of the work devoted to the active closed-loop control of instability waves in unexcited jets, ${ }^{1}$ we have demonstrated the possibility of active control of the installation noise of the unexcited jet.

At first step, the concept of noise control is formulated. The concept is based on the physical understanding of the low-frequency installation noise. As shown in many publications, ${ }^{6,9-11,13-17}$ the main mechanism of low-frequency noise intensification for jet-wing configuration is related to the scattering of the near field hydrodynamic pressure pulsations on the wing trailing edge. Moreover, if the trailing edge lies in the irrotational near field of the jet (i.e. it is not deeply inserted into the shear layer), the mechanism is linear. Near pressure field in the potential region close to the jet shear layer, in turn, is dominated by the linear instability waves. Thus, the mechanism of the installation noise is formulated in terms of linear scattering of linear instability waves by the wing trailing edge. Therefore, intensification or attenuation of instability waves by means of a control system should lead to the corresponding changes in the far-field noise for the jet-wing configuration.

At the second step of the research, a special set of test nozzles was prepared for the installation of HF DBD actuator inside the nozzle. Previous experience obtained during the ORINOCO project ${ }^{26}$ was used for the choice of the actuator's type. Ceramic nozzles, used in ORINOCO project, were replaced by more convenient metal nozzles with special ceramic coating. Such configuration of nozzle allowed using the body of the nozzle as the second electrode in the DBD arrangement. The second electrode was mounted close to the nozzle edge on the inner surface of the nozzle to ensure high effectiveness of instability waves excitation by the DBD actuator.

Then, a proof-of-concept study is performed. Relatively low-speed jet of Mach 0.4 is considered in the experiments together with a flat plate mimicking the wing. It is demonstrated that if installation noise is generated by instability waves artificially exited at a given frequency, it can be effectively amplified or attenuated, depending on the setting of the control system. The effect of control is clearly seen in the wide range of polar angles. The directivity of the scattered field is described by the analytical model of installation noise in all cases except the maximum attenuation. In the latter case, the scattered field becomes very weak so that it can hardly be identified on the background of the jet noise. At this stage, the parameters of the control system (amplitude and phase of the excitation) are adjusted manually.

At the final stage of the study, the automatic control system developed in Part I of the research ${ }^{1}$ is extended for the active closed-loop control of installation noise. The system is implemented on the LCARD module and realizes the control concept described by Belyaev et al. ${ }^{34}$ based on the on-line narrowband filtration of the reference signal and generation of the control action with phase compensation of the time delay arising in the feedback loop. The reference signal used for the control action generation is taken from the microphone flush mounted on the plate upstream from the plate trailing edge. Auxiliary near-field microphones are installed to monitor pressure fluctuations in the near field of the installed jet. It is shown that the plate changes the structure of the near field of the jet so as the axisymmetric mode starts to dominate close to the nozzle exit, in contrast to the uninstalled jet, for which the axisymmetric mode and the first azimuthal mode are of the same order. Such influence of the plate on the jet near field requires further investigation. It is demonstrated that proper settings of the control system allow to attenuate the signal on the reference microphone by about $2.5 \mathrm{~dB}$ in the frequency band defined by the digital filter implemented in the control module. On the frequencies slightly lower and higher than the central frequency of the filter, sidelobes of the signal amplification are observed. This effect is related to the phase characteristics of the digital filter and is discussed in Part I of the current study. ${ }^{1}$ It is demonstrated by means of the simple two-microphone azimuthal decomposition that the control action is focused on the axisymmetric mode only. This conclusion is confirmed by the analysis of the coherence functions. The control action leads to a significant drop in the coherence between the reference microphone and trailing edge microphone, and between the reference microphone and symmetric mode of the pressure field. 
Attenuation of the axisymmetric mode in the near field of the jet leads to a significant attenuation of the installation noise in the far field. The effectiveness of noise control at the target frequency is about $2.5 \mathrm{~dB}$ on the upstream microphones, and it decreases as the observer moves downstream. This is explained by the cardioid-like directivity of the installation noise, so that the influence of the control becomes less noticeable against the background jet noise. The coherence between the reference near-field microphone and far-field microphones reveals a distinct drop for all of the microphones except the one located at downstream observation angle and significantly dominated by the jet noise.

The results of the current work demonstrate real possibility to control far-field sound of the unexcited installed jet. The control action is realized in a narrow frequency band and has some penalty on the sides of the central frequency; nevertheless, it is, to our best knowledge, the first generally positive result of a closed-loop installation noise control. Further studies are required to clarify some not well-understood effects, to modify the control system in order to extend positive noise reduction effect for wider frequency band, and to investigate other jet operating conditions and jet-plate geometries.

\section{Acknowledgements}

The work has been supported by the Russian Ministry of Industry and Trade, project "ORINOCO-2" as a part of the European Union's Horizon 2020 project ARTEM, grant No 769350 (development of the installation noise control concept and its realization for excited jets) and by the Russian Foundation for Basic Research and Moscow city Government, project 19-31-70005 (realization of the concept for unexcited jets).

\section{References}

${ }^{1}$ O. Bychkov, G. Faranosov, V. Kopiev, Vl. Kopiev, I. Belyaev, I. Moralev, P. Kazansky. Plasma-based active closed-loop control of instability waves in unexcited turbulent jet. Part 1. Free jet. 25th AIAA/CEAS Aeroacoustics Conference, 2019.

${ }^{2}$ C.J. Mead, P.J.R. Strange. Under-wing installation effects on jet noise at sideline. AIAA Paper 98-2207, 1998.

${ }^{3}$ V.G. Mengle, L. Brusniak, R. Elkoby. Reducing Propulsion Airframe Aeroacoustic Interactions with Uniquely Tailored Chevrons: 3. Jet-Flap In-teraction. AIAA paper 2006-2435, 2006.

${ }^{4}$ V.G. Mengle. The Effect of Nozzle-to-Wing Gulley Height on Jet Flow Attachment to the Wing and Jet-Flap Interaction Noise. AIAA paper, AIAA-2011-2705, 2011.

${ }^{5}$ V.F. Kopiev, G.A. Faranosov, M.Yu. Zaytsev, E.V. Vlasov, R.K. Karavosov, I.V. Belyaev, N.N. Ostrikov. Intensification and suppression of jet noise sources in the vicinity of lifting surfaces. AIAA paper 2013-2284, 2013.

${ }^{6}$ A.V.G. Cavalieri, P. Jordan, W.R. Wolf, Y. Gervais. Scattering of wavepackets by a flat plate in the vicinity of a turbulent jet. Journal of Sound and Vibration. v. 333, pp. 6516-6531, 2014.

${ }^{7}$ V. Kopiev, I. Belyaev, G. Faranosov, Vl. Kopiev, N. Ostrikov, M. Zaytsev, G. Paranin. Numerical and Experimental Study of JFI Effect on Swept Wing. AIAA Paper 2014-3060, 2014.

${ }^{8}$ J. Lawrence. Aeroacoustic Interactions of Installed Subsonic Round Jets. PhD thesis, Univ. of Southampton, 2014.

${ }^{9}$ J. Vera, R.H. Self, M.J. Kinganz. The prediction of the radiated pressure spectrum produced by jet-wing interaction. AIAA Paper 2015-2216, 2015.

${ }^{10}$ S. Piantanida, V. Jaunet, J. Huber, W. Wolf, P. Jordan, A.V.G. Cavalieri. Scattering of turbulent-jet wavepackets by a swept trailing edge. AIAA Paper 2015-2998, 2015.

${ }^{11}$ O.P. Bychkov, G.A. Faranosov, S.L. Denisov, N.N. Ostrikov. Theoretical modeling of the excess noise due to jet-wing interaction. AIAA Paper 2016-2932, 2016.

${ }^{12}$ I.V. Belyaev, M.Yu. Zaytsev, V.F. Kopiev, N.N. Ostrikov, G.A. Faranosov. Studying the effect of flap angle on the noise of interaction of a high-bypass jet with a swept wing in a co-flow. Acoustical Physics, v. 63, N. 6, pp. 14-25, 2017.

${ }^{13}$ P.A.S. Nogueira, A.V.G. Cavalieri, P. Jordan. A model problem for sound radiation by an installed jet. Journal of Sound and Vibration, v. 391, pp. 95-115, 2017.

${ }^{14}$ B. Lyu, A.P. Dowling, I. Naqavi. Prediction of installed jet noise. Journal of Fluid Mechanics, v. 811, 2017, pp. $234-268$.

${ }^{15}$ O.P. Bychkov, G.A. Faranosov. An Experimental Study and Theoretical Simulation of Jet-Wing Interaction Noise. Acoustical Physics, v.64, N. 4, pp. 437-452, 2018.

${ }^{16}$ G.A. Faranosov, V.F. Kopiev, I.V. Belyaev, O.P. Bychkov, S.A. Chernyshev. On the Azimuthal Structure of Installed Jet Noise. AIAA paper AIAA 2017-3527, 2017.

${ }^{17}$ G.A. Faranosov, I.V. Belyaev, V.F. Kopiev, O.P. Bychkov. Azimuthal Structure of Low-Frequency Noise of Installed Jet. AIAA Journal, v. 57, N. 5, pp. 1885-1898, 2019.

${ }_{18}^{18}$ R.R. Mankbadi. Dynamics and Control Coherent Structures in Turbulent Jets. Applied Mechanics Reviews, v. 45, N. 6, pp. 219-248, 1992.

${ }^{19}$ M. Samimy, J.H. Kim, I. Adamovich, Y. Utkin, J. Kastner. Toward Noise Mitigation in High Speed and High Reynolds Number Jets Using Plasma Actuators. AIAA Paper 2006-2703, 2006.

${ }^{20}$ R. Maury, M. Koenig, L. Cattafesta, P. Jordan, J. Delville, J.-P. Bonnet, Y. Gervais. Extremum-seeking optimisation of fluidic jet-noise control. AIAA Paper 2009-3132, 2009. 
${ }^{21}$ M. Kearney-Fischer, J.H. Kim, M. Samimy. Noise control of a high Reynolds number high speed heated jet using plasma actuators. International Journal of Aeroacoustics, v. 10, N. 5-6, pp. 635-658, 2011.

${ }^{22}$ V.F. Kopiev, V.A., Bityurin I.V. Belyaev, S.M. Godin, M.Yu. Zaytsev, A.I. Klimov, V.A. Kopiev, I.A. Moralev, N.N. Ostrikov. Jet Noise Control using the Dielectric Barrier Discharge Plasma Actuators. Acoustical Physics, v. 58, N. 4, pp. 436-443, 2012.

${ }^{23}$ M. Samimy, M. Kearney-Fischer, J.H Kim. High-speed and high-Reynolds-number jet control using localized arc filament plasma actuators. Journal of Propulsion and Power, v. 28, N. 2, pp. 269-280, 2012.

${ }^{24}$ V.F. Kopiev, I.V. Belyaev, M.Yu. Zaytsev, V.A. Kopiev, G.A. Faranosov. Acoustic Control of Instability Waves in a Turbulent Jet. Acoustical Physics, v. 59, N. 1, pp.16-26, 2013.

${ }^{25}$ V.F. Kopiev, I.V. Belyaev, G.A. Faranosov, V.A. Kopiev, M.Yu. Zaytsev. Instability Wave Control in Turbulent Jet. AIAA Paper 2013-2085, 2013.

${ }^{26}$ V.F. Kopiev, Y.S. Akishev, I.V. Belyaev, N.K. Berezhetskaya, V.A. Bityurin, G.A. Faranosov, M.E. Grushin, A.I. Klimov, V.A. Kopiev, I.A. Kossyi, I.A. Moralev, N.N. Ostrikov, M.I. Taktakishvili, N.I. Trushkin, M.Yu. Zaytsev. Instability wave control in turbulent jet by plasma actuators. J. Phys. D: Appl. Phys., v. 47 / 5052012014, pp. 1-18, 2014.

${ }^{27}$ M. Crawley, A. Sinha, M. Samimy. Near-field and acoustic far-field response of a high-speed jet to excitation. AIAA Journal, v. 53, N. 7, pp. 1894-1909, 2015.

${ }^{28}$ M. Kœnig, K. Sasaki, A.V. Cavalieri, P. Jordan, Y. Gervais. Jet-noise control by fluidic injection from a rotating plug: linear and nonlinear sound-source mechanisms. J. Fluid Mech., v. 788, pp. 358-380, 2016.

${ }^{29}$ C.-W. Kuo, J. Cluts, M. Samimy. Exploring Physics and Control of Twin Supersonic Circular Jets. AIAA Journal, v. 55, N. 1, pp. $68-85,2017$.

${ }_{30}$ A. Sinha, A. Towne, T. Colonius, R.H. Schlinker, R. Reba, J.C. Simonich, D.W. Shannon. Active control of noise from hot supersonic jets. AIAA Journal, v. 56, N. 3, pp. 933-948, 2017.

${ }^{31}$ T. Suzuki, T. Colonius, Instability waves in a subsonic round jet detected using a near-field phased microphone array. J. Fluid Mech., v. 565, pp. 197-226, 2006.

${ }^{32}$ K. Gudmundsson, T. Colonius, Instability wave models for the near-field fluctuations of turbulent jets. J. Fluid Mech., v. 689 , pp. $97-128,2011$.

${ }_{33}$ P. Jordan, T. Colonius. Wave Packets and Turbulent Jet Noise. Ann. Rev. Fluid Mech., v. 45, 2013, pp.173-195.

${ }^{34}$ I.V. Belyaev, O.P. Bychkov, M.Y. Zaitsev, V.A. Kopiev, V.F. Kopiev, N.N. Ostrikov, G.A. Faranosov, S.A. Chernyshev. Development of the Strategy of Active Control of Instability Waves in Unexcited Turbulent Jet. Fluid Dynamics, v. 53, N. 3, pp. 347-360, 2018.

${ }^{35}$ R.M. Munt. The interaction of sound with a subsonic jet issuing from a semi-infinite cylindrical pipe. J. Fluid Mech., v. 83, N. 4, pp. 609-640, 1977.

${ }^{36}$ R.E.A. Arndt, D.F. Long, M.N. Glauser. The proper orthogonal decomposition of pressure fluctuations surrounding a turbulent jet. J. Fluid Mech., v. 340, pp.1-33, 1997. 